\title{
Democratic therapeutic community treatment for personality disorder: randomised controlled trial
}

Steve Pearce, Lisle Scott, Gillian Attwood, Kate Saunders, Madeleine Dean, Ritz De Ridder, David Galea, Haroula Konstantinidou and Mike Crawford

\section{Background}

Democratic therapeutic community (DTC) treatment has been used for many years in an effort to help people with personality disorder. High-quality evidence from randomised controlled trials (RCTs) is absent.

\section{Aims \\ To test whether DTC treatment reduces use of in-patient services and improves the mental health of people with personality disorder.}

\section{Method}

An RCT of 70 people meeting DSM-IV criteria for personality disorder (trial registration: ISRCTN57363317). The intervention was DTC and the control condition was crisis planning plus treatment as usual (TAU). The primary outcome was days of in-patient psychiatric treatment. Secondary outcomes were social function, mental health status, self-harm and aggression, attendance at emergency departments and primary care, and satisfaction with care.
All outcomes were measured at 12 and 24 months after randomisation.

\section{Results}

Number of in-patient days at follow-up was low among al participants and there was no difference between groups. At 24 months, self- and other directed aggression and satisfaction with care were significantly improved in the DTC compared with the TAU group.

\section{Conclusions}

DTC is more effective than TAU in improving outcomes in personality disorder. Further studies are required to confirm this conclusion.

\section{Declaration of interest}

None.

\section{Copyright and usage}

(c) The Royal College of Psychiatrists 2017.
Personality disorders are common conditions ${ }^{1}$ with high morbidity, ${ }^{2}$ high unmet needs ${ }^{3}$ and high healthcare costs. ${ }^{4}$ People with personality disorders are at higher risk of other mental disorders, substance misuse and suicide. ${ }^{5,6}$ Concerns have been expressed about the quality of services for people with personality disorder, ${ }^{7}$ and the evidence base for the effectiveness of interventions is weak. ${ }^{8,9}$ Evidence for effective treatment of borderline personality disorder remains sparse. To date there is little evidence for the efficacy of psychopharmacological treatments. ${ }^{10}$ Among psychosocial treatments, dialectical behaviour therapy has demonstrated a beneficial effect in several randomised trials at more than one centre. ${ }^{11}$ Mentalisation-based therapy has demonstrated beneficial effects when delivered as part of a day programme ${ }^{12}$ or out-patient weekly individual and group psychotherapy. ${ }^{13}$ Novel approaches such as dialectical behaviour therapy and mentalisation-based therapy may be replacing more established approaches to the treatment of borderline personality disorder.

In the UK one of the most widespread interventions has been democratic therapeutic communities (DTC), which have provided specialist services for people with personality disorders for over 50 years. ${ }^{14}$ DTC is a form of psychosocial treatment based on a collaborative and deinstitutionalised approach to staff-patient interaction; particular emphasis is placed on empowerment, personal responsibility, shared decision-making and participation in communal activity. ${ }^{15}$ Much background work has been completed that paves the way for an experimental evaluation of DTC: the mechanism of action of the intervention has been extensively studied ${ }^{16,17}$ and observational evidence for possible benefits of the intervention have been established. ${ }^{18-20}$ Although the limitations of such studies are widely acknowledged, highquality randomised controlled trials (RCTs) have not been conducted. A systematic review concluded that such studies were now required, ${ }^{21}$ a conclusion in keeping with recommendations for the evaluation of complex interventions. ${ }^{22}$ We report here the results of the first RCT of DTC treatment for people with personality disorder, the Therapeutic Community Intervention Trial (TaCIT, trial registration: ISRCTN57363317).

\section{Method}

\section{Trial design}

Participants were recruited from referrals to an established personality disorder service. The study was approved by the local National Health Service ethics committee in Oxford, ethics number 08/H0605/87. Informed consent was obtained from all participants. The study protocol and consent forms were discussed in all four therapeutic communities in which participants were to be treated in order to obtain consent from the communities for potential study participants to participate in their therapy. This was done because of the uniquely consent-based nature of the DTC intervention, in which any changes to treatment or the running of the community is discussed with the members. The three trial assessors (G.A., S.P., L.S.) were all clinicians experienced in working with personality disorder, and trained in the use of the Structured Clinical Interview for Axis II disorder (SCID-II), ${ }^{23}$ which was used to establish personality disorders diagnosis. Comorbid mental illness was assessed independently from a review of medical records. After consent was obtained and personality disorders status confirmed, baseline measures were collected by the assessor. Participants were then seen between 2 and 4 weeks later and their randomisation status communicated to them. Those randomised to DTC were immediately put on the waiting list for the preparatory DTC groups, and those randomised to TAU received their first session of crisis planning. 
Joint crisis planning was chosen as the TAU condition as it is valued by patients, but may have little impact on patient outcomes. $^{24}$

\section{Recruitment and randomisation}

Participation in the trial was offered to all patients referred to the Oxfordshire Complex Needs Service who were allocated to one of the trial assessors and met inclusion criteria. Inclusion criteria were: resident in the catchment area of the personality disorder service; aged between 16 and 65; and having a diagnosis of personality disorder, assessed using the SCID-II. ${ }^{23}$ Exclusion criteria mirrored those commonly used in clinical practice when offering DTC treatment: (a) a primary diagnosis of a psychotic disorder, alcohol or drug dependence (those with a history of transient psychotic symptoms and non-dependent substance misuse were included); (b) a degree of learning disability, or intellectual impairment, that prevents engagement in DTC services. Independent remote randomisation was conducted at the Centre for Mental Health, Imperial College London. We used a computer-generated random scheme to allocate participants to DTC and TAU in a ratio of $1: 1$. We used stratification to balance potential confounding variables (age - above or below 30, gender and baseline service utilisation - previous history of admission to a mental health unit or presentation to an emergency department in crisis) across study groups. The local DTC and community mental health team were then informed of the patient's randomisation status.

\section{Interventions}

\section{DTC treatment}

Participants randomised to DTC entered the normal treatment process for patients entering DTC treatment in the Oxfordshire Complex Needs Service. This consists initially of attendance at a DTC preparatory group meeting weekly for $2 \mathrm{~h}$ per week, for up to a year. The DTC preparatory group incorporates the core elements of DTC practice described below in a brief format. After a minimum of 3 months' attendance at this group, participants are able join the DTC via a democratic selection process in which current members and staff vote. Four DTCs hosted participants in the study, based around Oxfordshire. DTCs ranged in size from 14 to 18 members. Participants received DTC therapy for a maximum of 18 months. DTC treatment consisted of between 5 and $15 \mathrm{~h}$ per week of mixed structured and unstructured group therapy adhering to the following DTC principles.

(a) Democratisation: shared decision-making around group matters, when necessary involving transparent voting procedures. Staff retain responsibility for maintaining safe and effective treatment. Members chair and record meetings, and make decisions that have substantive effects on the way the community runs. This has the effect of promoting responsible agency. ${ }^{17}$

(b) Permissiveness: a wide range of behaviour is tolerated, as long as it does not harm another member or impede another member's treatment. Behaviour is understood and discussed rather than condemned or forbidden. The principle of responsibility without blame ${ }^{25}$ is used to inform this process.

(c) Reality confrontation: members and staff challenge one another around behaviour and attitudes, and feedback to one another about their impact. This is done in a compassionate rather than judgemental manner.

(d) Communalism: there is an element of shared living in DTC. Staff and members eat together, undertake tasks together and share leisure activities as far as is allowed by the programme. During such informal group activity staff and members practice authenticity with one another. Situations that arise in the shared life of the community (the milieu) are used by staff and members to inform the process in the formal groups, a process known as the 'living learning experience'. These four principles were noted by Rapoport in 1960 at Belmont Hospital. ${ }^{26}$

(e) A culture of enquiry. ${ }^{27}$ All events in the DTC are available for consideration by the members and staff, and a questioning attitude is encouraged.

(f) Milieu approach/a therapeutic environment. ${ }^{28}$ In addition to informal interactions informing group process, the entirety of the community atmosphere and activity, including the involvement of members in administrative tasks, is held to be therapeutic.

The DTCs taking part in this study were members of the Association of Therapeutic Communities. The service is accredited by the Royal College of Psychiatrists Centre for Quality Improvement. ${ }^{16}$

\section{Treatment as usual}

Participants randomised to TAU were offered three sessions of joint crisis planning by the clinician who assessed them. This consisted of the collaborative construction of a crisis plan identifying triggers for deterioration in mental state, and practical steps that can be taken to get support or to maintain stability. Other elements of TAU varied depending on patient needs and local service organisation but included out-patient monitoring, consideration of psychotropic medication, and the option of referral to out-patient psychotherapy treatment and in-patient psychiatric treatment at times of crisis. These elements were delivered by local primary care services or community mental health teams.

\section{Baseline and outcome measures}

Eligibility was assessed by examining case notes for clinical diagnosis and completion of the SCID-II, ${ }^{23}$ the Fast Alcohol Screening Test, ${ }^{29}$ and Drug Abuse Screening Test. ${ }^{30}$ For those who met inclusion criteria, data collection on baseline measures was completed prior to randomisation, using an examination of paper and electronic medical records to quantify use of in-patient psychiatric services (primary outcome) and contacts with emergency medical services in the previous 6 months, and patient interview using:

(a) the 12-item General Health Questionnaire - a measure of general mental health; ${ }^{31}$

(b) the Social Functioning Questionnaire - an eight-item validated measure of social functioning that is sensitive to change; ${ }^{32}$

(c) extent of any self-harm or aggressive behaviour towards others during the preceding 4 weeks using the Modified Overt Aggression Scale; ${ }^{33}$

(d) satisfaction with care using the eight-item Client Satisfaction Questionnaire; $^{34}$

(e) frequency of suicidal acts and acts of self-harm collected via a self-report questionnaire developed specifically for the study;

(f) utilisation data on visits to primary care and emergency departments were collected via a self-report questionnaire developed specifically for the study. 
Follow-up interviews were conducted 12 and 24 months after randomisation using the measures (a) to (f) listed above combined with an examination of hospital records to obtain information on psychiatric admissions. Rater masking was maintained by specific instructions to participants and clinical teams not to disclose treatment details. Patient data were held securely and all personal identifiers removed, with randomisation details held separately and password protected. Patients completing follow-up interviews were offered a $£ 25$ honorarium in recognition of any inconvenience caused to them by participation in the study. Researchers were asked to state if they believed they had become aware of a participant's allocation status in order to monitor the extent to which rater masking was maintained.

\section{Data analysis}

The sample size estimate was based on our primary outcome. A previous observational study found that patients treated in DTCs have a mean number of 45 days (s.d.=71) of in-patient psychiatric treatment in the year prior to referral and 12 days (s.d.=22) in the year following referral. ${ }^{19}$ A sample of 76 patients (38 DTC and $38 \mathrm{TAU}$ ) would be required to have $80 \%$ power and 5\% level of statistical significance to demonstrate a reduction in the mean number of in-patient days of this magnitude. As information on use of in-patient psychiatric services was to be extracted from hospital records we estimated that loss to follow-up would be low (10\%) and therefore aimed to randomise 85 people into the trial. We conducted a complete-case analysis according to the treatment to which participants were randomised (intention-to-treat). We used descriptive statistics to examine baseline characteristics of study participants. For continuous variables we present the number of observations $(n)$, with either the mean and standard deviation, for normally distributed variables, or the median and interquartile range (IQR) for non-normally distributed variables. For categorical variables we used counts and percentages. We then calculated differences in outcomes between baseline and 12 and 24 months using appropriate univariate statistics. Finally, we examined differences in outcomes between those randomised to DTC and TAU adjusted for baseline level, age and gender using binary logistic regression for categorical variables and linear regression for normally distributed continuous variables.

\section{Results}

Study recruitment commenced in February 2009. Between February 2009 and November 2012, 121 people were assessed for participation in the study. Of these $70(57.9 \%)$ were eligible and were randomised. Reasons for non-participation were: unwilling to provide consent $(n=34,27.0 \%)$; no personality disorder $(n=15,12.4 \%)$; and comorbid dependence on alcohol or drugs $(n=5,4.1 \%)$. One person was excluded because they had a primary diagnosis of psychosis (see flow diagram - Fig. 1). Age, gender and ethnicity of those who did and did not take part in the study are presented in Table 1 . Of the 70 people who took part in the study, equal numbers $(n=35)$ were allocated to each arm of the trial. Sociodemographic and clinical characteristics of participants by study group are presented in Table 2 .

\section{Flow of participants through the trial}

The CONSORT diagram (Fig. 1) summarises the flow of participants through the trial. Routine data on attendance at emergency medical services and admission to hospital were obtained for all 70 participants. Data on secondary outcomes from interviews with participants were obtained from 45 (64.3\%)

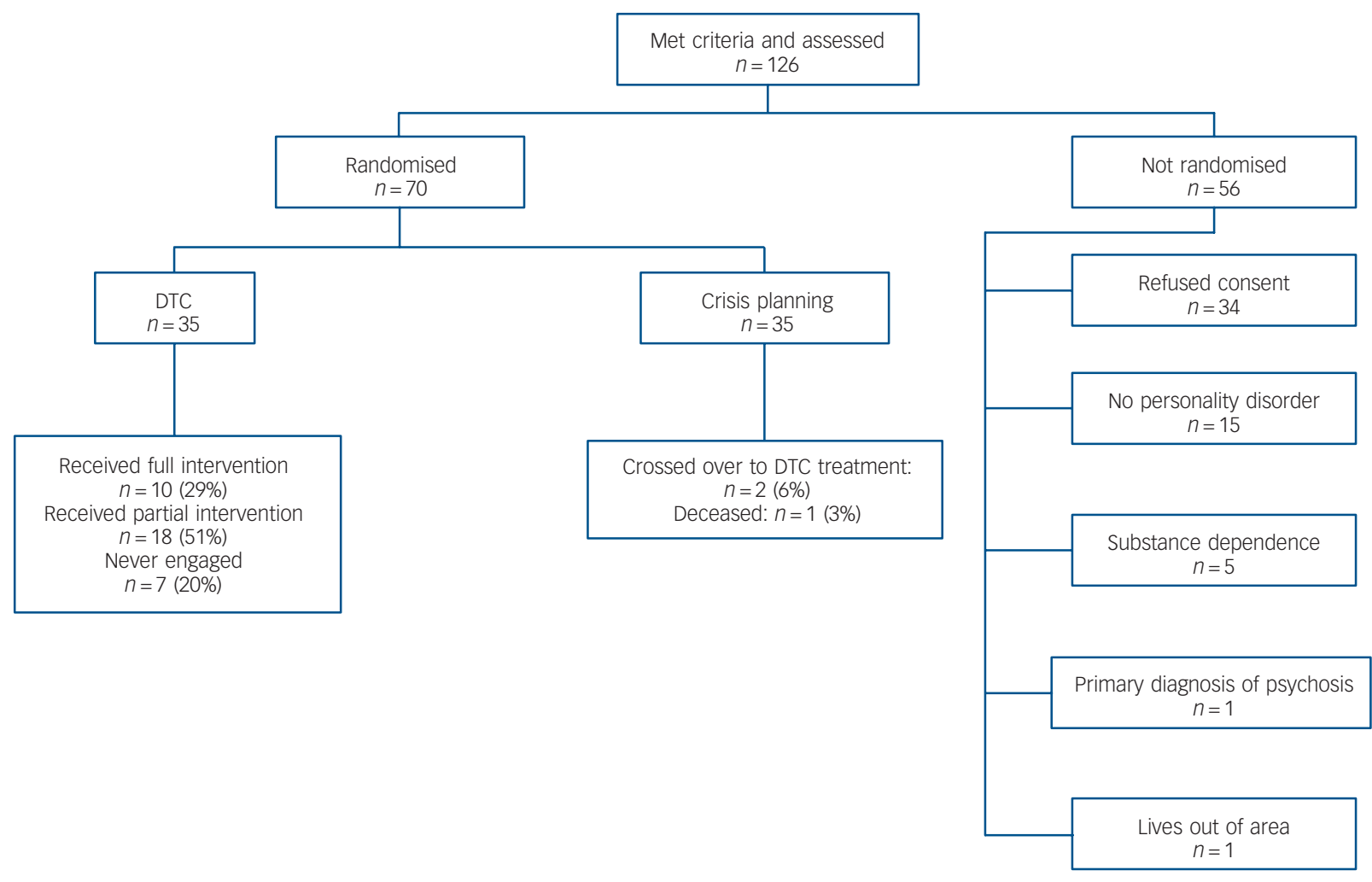

Fig. 1 Study flow chart at 2-year follow-up. 


\begin{tabular}{llcl|}
\hline $\begin{array}{l}\text { Table } 1 \\
\text { Variable }\end{array}$ & Age, gender and ethnicity of participants and non-participants & & Total $(n=121)$ \\
\hline $\begin{array}{l}\text { Gender, } n(\%) \\
\text { Women }\end{array}$ & Participants $(n=70)$ & $33(64.7)$ & $88(72.7)$ \\
Men & $55(78.6)$ & $18(35.3)$ & $33(27.3)$ \\
\hline Age, mean (IQR) & $15(21.4)$ & $31.37(9.54)$ & $32.91(10.17)$ \\
\hline $\begin{array}{l}\text { Ethnicity, } n \text { (\%) } \\
\text { White British }\end{array}$ & $34.03(10.54)$ & $45(88.2)$ & $114(94.2)$ \\
White other & $69(98.6)$ & $3(5.9)$ & $4(3.3)$ \\
Black and minority ethnic & $1(1.4)$ & $3(5.9)$ & $3(2.5)$ \\
\hline
\end{tabular}

\begin{tabular}{|c|c|c|c|c|}
\hline Variable & Control $(n=35)$ & Intervention $(n=35)$ & Total $(n=70)$ & Total, $n$ \\
\hline Gender, $n(\%)$ & & & & 70 \\
\hline Women & $8(22.9)$ & $7(20.0)$ & $15(21.4)$ & \\
\hline Men & $27(77.1)$ & $28(80.0)$ & $55(78.6)$ & \\
\hline Age, mean (s.d.) & $33.4(11.2)$ & $34.6(10.0)$ & $34.0(10.5)$ & 70 \\
\hline Ethnicity, $n(\%)$ & & & & 70 \\
\hline White British & $35(100.0)$ & $34(97.1)$ & $69(98.6)$ & \\
\hline White other & $0(0.0)$ & $1(2.9)$ & $1(1.4)$ & \\
\hline Number of personality disorder diagnoses, median (IQR) & $2(1-3)$ & $3(2-4)$ & $3(2-4)$ & 70 \\
\hline Type of personality disorder, $n$ (\%) & & & & 70 \\
\hline Avoidant & $21(60.0)$ & $26(74.3)$ & $47(67.1)$ & \\
\hline Dependent & $6(17.1)$ & $8(22.9)$ & $14(20.0)$ & \\
\hline Obsessive-compulsive & $2(5.7)$ & $9(25.7)$ & $11(15.7)$ & \\
\hline Paranoid & $9(25.7)$ & $16(45.7)$ & $25(35.7)$ & \\
\hline Schizotypal & $0(-)$ & $1(2.9)$ & $1(1.4)$ & \\
\hline Schizoid & $0(-)$ & $0(-)$ & $0(-)$ & \\
\hline Histrionic & $5(14.3)$ & $2(5.7)$ & $7(10.0)$ & \\
\hline Narcissistic & $7(20.0)$ & $5(14.3)$ & $12(17.1)$ & \\
\hline Borderline & $32(91.4)$ & $33(94.3)$ & $65(92.9)$ & \\
\hline Antisocial & $2(5.7)$ & $5(14.3)$ & $7(10.0)$ & \\
\hline Axis I diagnoses & & & & 70 \\
\hline Major depressive disorder & $16(45.7)$ & $17(48.6)$ & $33(47.1)$ & \\
\hline Any affective disorder & $18(51.4)$ & $19(54.3)$ & $37(52.9)$ & \\
\hline Any anxiety disorder & $4(11.4)$ & $5(14.3)$ & $9(12.9)$ & \\
\hline Primary outcome: days of in-patient treatment, median (IQR) & $0(0-1)$ & $0(0-1)$ & $0(0-1)$ & 70 \\
\hline \multicolumn{5}{|l|}{ Secondary outcomes } \\
\hline Any in-patient admission, $n(\%)$ & $9(25.7)$ & $9(25.7)$ & $18(25.7)$ & 70 \\
\hline Number of attendances at emergency departments, median (IQR) & $1(0-2)$ & $1(0-2)$ & $1(0-2)$ & 70 \\
\hline Any attendance at emergency departments, $n(\%)$ & $20(57.1)$ & $19(54.3)$ & $39(55.7)$ & 70 \\
\hline Number of general practice attendances, median (IQR) & $15(7.5-20)$ & $10(5-20)$ & $12(6-20)$ & 65 \\
\hline Social Functioning, mean (s.d.) & $17.4(4.1)$ & $17.2(4.5)$ & $17.3(4.3)$ & 66 \\
\hline General Health Questionnaire, mean (s.d.) & $8.8(3.6)$ & $9.3(3.2)$ & $9.1(3.4)$ & 68 \\
\hline Total Modified Overt Aggression Scale score, median (IQR) & $3(0-5)$ & $3(1-5)$ & $3(1-5)$ & 63 \\
\hline Acts of self-harm, any (\%) & $21(65.6)$ & $21(67.7)$ & $42(66.7)$ & 63 \\
\hline Number of acts, median (IQR) & $2(0-10)$ & $3(0-20)$ & $2(0-11)$ & 63 \\
\hline Client satisfaction, mean (s.d.) & $21.5(5.9)$ & $21.1(5.9)$ & $21.3(5.9)$ & 63 \\
\hline
\end{tabular}

participants at 12 months and $38(54.3 \%)$ at 24 months. Of the 32 (45.7\%) participants who were not followed up at 2 years, 1 died, 5 formally withdrew from the study and the other 26 either could not be traced or did not take up repeated offers to be assessed through letters and phone calls. Two participants asked to cross over from the TAU arm of the study to the DTC arm, one of whom also formally withdrew.

\section{Uptake of allocated treatments}

In total, 35 participants were allocated to the DTC intervention, of whom $28(80 \%)$ attended at least one preparatory DTC group. Of those who attended a preparatory group the mean attendance was 46 weeks (range 2 to 75 ) and $21(60 \%)$ were still attending the programme at 12 months. Ten $(29 \%)$ of those in the active arm of the trial also attended sessions on the DTC programme. Mean length of attendance was 47 weeks and all were still attending the programme when 12-month follow-up data were collected.

\section{Main and secondary outcomes}

Primary and secondary outcomes at 12- and 24-month follow-up are presented in Tables 3 and 4, respectively. Although small numbers in both arms of the trial presented to emergency medical services and were admitted to hospital, none of these was judged to be a serious adverse reaction related to study treatment. In both arms of the trial the median number of in-patient days was nil. Researchers reported five occasions when they became aware of a participants' allocation status at 12-month follow-up, and three occasions at 24-month follow-up. Although fewer people in the active intervention arm had an admission to hospital 12 months after randomisation, the difference was not statistically significant (difference $11.4 \%, 95 \%$ CI -10.1 to $31.6 \%$ ). DTC showed 

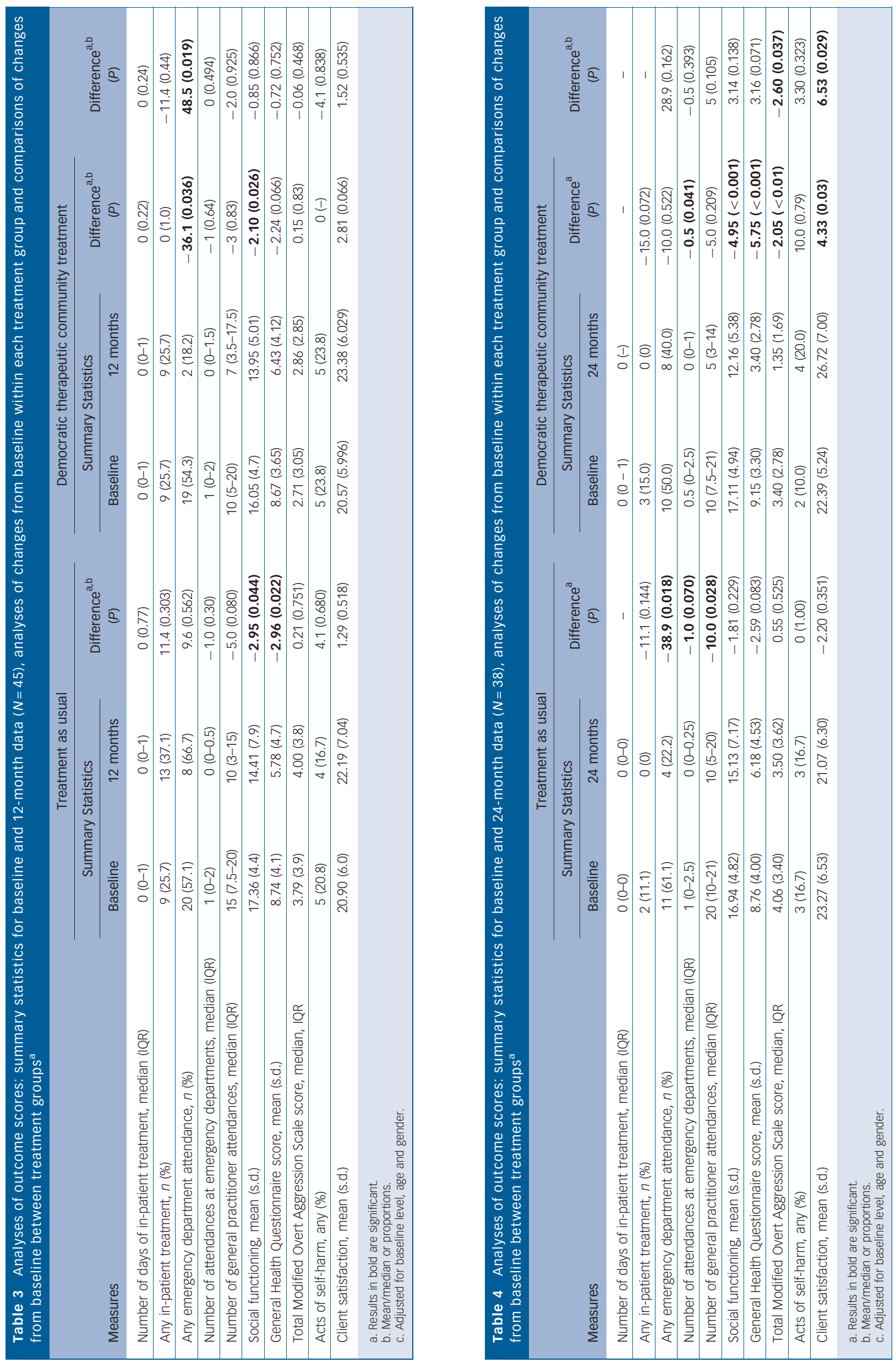
significant advantages over TAU in aggression and self-harm measured by the Modified Overt Aggression Scale, and satisfaction with treatment, measured by the Client Satisfaction Questionnaire. There were no significant differences in other outcomes between those randomised to DTC and TAU.

Although participants receiving DTC showed a significant improvement in social functioning (Social Function Questionnaire) and mental health (General Health Questionnaire), participants receiving TAU showed a non-significant improvement on these measures, and the difference between conditions was not statistically significant. Online Fig. DS2 illustrates these results from Tables 3 and 4 in graphical form.

\section{Discussion}

Although concerns have been raised about the feasibility of experimental studies of DTC treatment, ${ }^{35,36}$ we have shown that an RCT of DTC treatment is possible. Therapeutic communities evolve and change according to the wishes of their members. This and the fact that DTC is a milieu-based intervention make it difficult to devise an adherence instrument. We addressed the problem of ensuring adherence to the model through service accreditation. ${ }^{37,38}$ Accreditation ensures adherence to the core processes and values of DTC practice and involves a detailed examination of the technique and environment. ${ }^{39}$ The trial failed to support the primary hypothesis, that the active treatment would reduce use of in-patient services more than TAU. Levels of use of in-patient psychiatric services were lower among all participants in the study compared with those reported in the previous observational study we used for the sample size calculation. ${ }^{19}$ It is possible that this reflects differences in severity of personality disorder among people in the previous study, which was based in an in-patient therapeutic community, and changes in the organisation and delivery of acute care in the UK during the intervening period. Greater emphasis is now placed upon providing intensive community support at times of crisis in an attempt to reduce use of in-patient services. As all participants in the study had low levels of in-patient mental health treatment we were unable to properly explore the impact of the intervention on this outcome. Caution needs to be exercised in interpreting differences in secondary outcomes in the study because of loss to follow-up. The results of the complete-case analysis suggest that DTC is superior to TAU in reducing self-harm, violence and aggression, and improving satisfaction with care.

Following randomisation, treatment received by study participants receiving DTC was identical to that received by patients receiving DTC treatment who were not taking part in the study. By ensuring that the treatment received by the study participants reflects precisely the treatment routinely available we aimed to increase the generalisability of the study findings. The service was oversubscribed during the study period, so there was a waiting time prior to joining the DTC preparatory group. For this reason, by the 24-month follow-up point, just under a third (29\%) of participants received full DTC treatment, and $80 \%$ received DTC preparatory work. The preparatory groups run as brief DTCs, and include all the major elements of the DTC intervention. As a result of the emphasis on empowerment in DTC treatment, transparency and involvement of members is promoted. This raises concerns that a randomised trial, which although based on consent involves decision-making about access to treatment (in this case randomised allocation) that is administered by a professional, might damage the autonomy of the members of a DTC participating in the trial. We addressed this first by obtaining consent from the participating therapeutic community, as well as individual participants, and second by measuring elements of group function in the therapeutic communities involved in the trial, and comparing it with the functioning of two DTCs in a neighbouring county. Autrique et $a l^{40}$ compared the DTCs involved in the TaCIT trial with two DTCs not involved on the Community Oriented Environment Scale, ${ }^{41}$ and did not find any deleterious effects on therapeutic culture.

\section{Strengths and limitations of the study}

The study has a number of strengths. It is an effectiveness study of patients referred to a real-world personality disorder service subject to the normal difficulties and pressures involved in the routine delivery of mental healthcare, including delays in access to treatment. As in other specialist treatment services for people with personality disorder, most study participants had more than one personality disorder and most had comorbid Axis I mental disorders. ${ }^{13,42}$ There is a well characterised control group, who did not differ significantly from the intervention group. There were very few exclusion criteria, and the levels of comorbidity were high, which mirrors normal clinical practice. ${ }^{2}$ Personality disorders diagnosis was made using a formal interview (the SCID-II). The DTC intervention is well established, widely available ${ }^{21,43}$ and well described. ${ }^{16}$ The study is of treatment of any DSM-IV personality disorder, and borderline, avoidant and paranoid personality disorder were all present in over a third of participants. However, the fact that over $90 \%$ had a DSM-IV borderline personality disorder may limit the applicability of the conclusions to patient groups with personality disorder without comorbid borderline personality disorders.

The small sample size limits the power of the study to detect effects of the intervention. The participants lost to follow-up at both 12- and 24-month follow-up is high. Although characteristics of those who were and were not followed up are similar, caution needs to be exercised when interpreting the results of the study. The study was unfunded, and all masked raters were volunteers, who moved on regularly; this is likely to be one of the reasons for the low rate of follow-up. Four out of five participants attended at least one DTC preparatory group, for an average of almost a year (46 weekly sessions). A total of $29 \%$ of participants had received full DTC treatment by the 24-month follow-up point, a level likely to have been influenced by waiting times both to start treatment in the DTC preparatory group, and the 3 - to 12-month period spent in the preparatory group prior to treatment in the DTC proper. In total, $80 \%$ received a weekly DTC intervention (the preparatory group). These groups incorporate the essential elements of DTC, but at lower intensity.

In order to address the limitations of the study, a longer follow-up period is required. Personality disorders are long-term conditions, and trials with short follow-ups have been criticised. ${ }^{9}$ At 2 years, this trial demonstrates a benefit at longer follow-up than most. ${ }^{9}$ The authors are currently carrying out a 5 -year follow-up study. A larger trial would address the possibility that this study was too small to detect all the potential effects, and future work should be multicentre to demonstrate the effectiveness of the intervention delivered by different teams. Although this study has found evidence of clinical effectiveness, future research should examine the costs and cost-effectiveness of this treatment approach.

\section{Implications}

DTCs place an emphasis on peer support and challenge, and Mahlke \& Bock $^{44}$ found an effect for peer support in a range of mental disorders including personality disorders in a randomised 
trial. The promotion of belongingness, which is likely to be a substantial feature of integrated treatments for personality disorder, has an independent effect on well-being and mental health ${ }^{45}$ and is prominent in DTC. ${ }^{17}$ Similarly, the promotion of responsible agency has beneficial effects on impulsivity, self-efficacy and the ability to make good choices. ${ }^{17}$

Other elements of DTC functioning are likely to have benefits that are less easy to quantify. The National Institute for Health and Care Excellence recommends that in borderline personality disorder 'Specialist personality disorder services should involve people with personality disorders and families or carers in planning service developments, and in developing information about services ... people with personality disorders may also provide services, such as training for professionals, education for service users and families or carers, and facilitating peer support groups. ${ }^{46}$ Therapeutic communities base their practice on the assumption that patients will be fully involved not only in planning service developments, but also in planning the details of their own care, and encourages members to act in a mentoring capacity to other members. In the DTCs involved in this trial, members and ex-members are involved in recruiting and training professionals, facilitating groups, producing literature about the service and providing carer education. This is why the consent of the four therapeutic communities was sought for their participation in the trial, in addition to individual consent from the participants.

This trial is the first randomised study of DCT treatment for people with personality disorder. It provides preliminary data demonstrating beneficial effects compared with a control condition at 2-year follow-up. We have also demonstrated that it is possible to overcome the obstacles previously identified to carrying out an RCT of therapeutic community treatment. It is thus now possible to build on these results with a longer-term follow-up study to confirm the sustainability of these benefits, which is underway, and a larger multicentre trial of DTC treatment for personality disorder, which is now required to confirm these results.

Steve Pearce, MRCPsych, Lisle Scott, MSC, MBChB, Gillian Attwood, RMN Oxford Health NHS Foundation Trust, Oxford, UK; Kate Saunders, DPhil, MRCPsych, University of Oxford, Oxford; Madeleine Dean, MSc, School of Health and Human Sciences, University of Essex, Colchester, UK; Ritz De Ridder, MRCPsych, Berkshire Healthcare NHS Foundation Trust, Bracknell, UK; David Galea, MRCPsych, Seksjon for psykosebehandling, Oslo Universitetssykehus HF, Oslo, Norway; Haroula Konstantinidou, MRCPsych, Francis Dixon Lodge, Leicester; Mike Crawford, MD, Konstantinidou, MRCPSych, Francis Dixon Lodge,
FRCPsych, Imperial College London, London, UK.

Correspondence: Steve Pearce, Oxford Health NHS Foundation Trust, Manzil Way, Oxford, OX4 1XE, UK. Email: steve.pearce@oxfordhealth.nhs.uk

First received 24 Mar 2016, final revision 29 Jul 2016, accepted 30 Jul 2016

\section{Acknowledgements}

M.C. and K.S. are supported by the National Institute for Health Research (NIHR) Biomedica Research Centre based at Imperial College Healthcare NHS Trust and Imperial College London. The views expressed are those of the authors and not necessarily those of the NHS, NIHR or the Department of Health. K.D. is supported by a Wellcome Strategic Award Collaborative Network in Bipolar Disorder to Improve Outcomes and a NARSAD Young Investigator Award.

\section{References}

1 Coid J, Yang M, Tyrer $\mathrm{P}$, Roberts $\mathrm{A}$, Ullrich S. Prevalence and correlates of personality disorder in Great Britain. Br J Psychiatry 2006; 188: 423-31.

2 Skodol AE, Gunderson JG, McGlashan TH, Dyck IR, Stout RL, Bender DS, et al. Functional impairment in patients with schizotypal, borderline, avoidant, or obsessive-compulsive personality disorder. Am J Psychiatry 2002; 159: 276.

3 Hayward M, Slade M, Moran P. Personality disorders and unmet needs among psychiatric inpatients. Psychiatr Serv 2006; 57: 538-43.
4 Bender DS, Skodol AE, Pagano ME, Dyck IR, Grilo CM, Shea MT, et al. Prospective assessment of treatment use by patients with personality disorders. Psychiatr Serv 2006; 57: 254-7.

5 Pompili M, Girardi $P$, Ruberto A, Tatarelli R. Suicide in borderline personality disorder: a meta-analysis. Nord J Psychiatry 2005; 59: 319-24.

6 Zanarini MC, Frankenburg FR, Dubo ED, Sickel AE, Trikha A, Levin A, et al. Axis I comorbidity of borderline personality disorder. Am J Psychiatry 1998; 155: 1733-9.

7 Crawford MJ, Price K, Rutter D, Moran P, Tyrer P, Bateman A, et al. Dedicated community-based services for adults with personality disorder: Delphi study. Br J Psychiatry 2008; 193: 342-3.

8 Bateman AW, Gunderson J, Mulder R. Treatment of personality disorder. Lancet 2015; 385: 735-43.

9 Levy KN. Psychotherapies and lasting change. Am J Psychiatry 2008; 165: $556-9$.

10 Lieb K, Völlm B, Rücker G, Timmer A, Stoffers JM. Pharmacotherapy for borderline personality disorder: Cochrane systematic review of randomised trials. Br J Psychiatry 2010; 196: 4-12.

11 Kliem S, Kroger C, Kosfelder J. Dialectical behavior therapy for borderline personality disorder: a meta-analysis using mixed-effects modeling. J Consult Clin Psychol 2010; 78: 936.

12 Bateman A, Fonagy P. 8-year follow-up of patients treated for borderline personality disorder: mentalization-based treatment versus treatment as usual. Am J Psychiatry 2008; 165: 631-8.

13 Bateman A, Fonagy P. Randomized controlled trial of outpatient mentalization-based treatment versus structured clinical management for borderline personality disorder. Am J Psychiatry 2009; 166: 1355-64.

14 Whiteley S. The evolution of the therapeutic community. Psychiatr Q 2004; 75: $233-48$

15 Campling P. Therapeutic communities. Adv Psychiatr Treat 2001; 7: 365-72.

16 Hirst O, Paget S. Service Standards for Therapeutic Communities. Royal College of Psychiatrists, College Research Unit, 2005.

17 Pearce S, Pickard $\mathrm{H}$. How therapeutic communities work: specific factors related to positive outcome. Int J Soc Psychiatry 2013; 59: 636-45.

18 Dolan B, Warren F, Norton K. Change in borderline symptoms one year after therapeutic community treatment for severe personality disorder. Br J Psychiatry 1997; 171: 274-9.

19 Davies S, Campling P. Therapeutic community treatment of personality disorder: service use and mortality over 3 years' follow-up. Br J Psychiatry 2003; 182: s24-7.

20 Chiesa M, Fonagy P, Holmes J, Drahorad C. Residential versus community treatment of personality disorders: a comparative study of three treatment programs. Am J Psychiatry 2004; 161: 1463-70.

21 Lees J, Manning N, Rawlings B. Therapeutic Community Effectiveness: A Systematic International Review of Therapeutic Community Treatment for People with Personality Disorders and Mentally Disordered Offenders. University of York, 1999.

22 Craig P, Dieppe P, Macintyre S, Michie S, Nazareth I, Petticrew M. Developing and evaluating complex interventions: the new Medical Research Council guidance. BMJ 2008; 337: a1655.

23 First MB, Spitzer RL, Gibbon M, Williams JBW. Structured Clinical Interview for DSM-IV Personality Disorders, (SCID-II). American Psychiatric Press, 1997.

24 Borschmann R, Barrett B, Hellier JM, Byford S, Henderson C, Rose D, et al Joint crisis plans for people with borderline personality disorder: feasibility and outcomes in a randomised controlled trial. Br J Psychiatry 2013; 202: 357-64.

25 Pickard H. Responsibility without blame: empathy and the effective treatment of personality disorder. Philos Psychiatr Psychol 2011; 18: 209-24.

26 Rapoport R. Community as Doctor. Tavistock Publications, 1960.

27 Main T. The concept of the therapeutic community: variations and vicissitudes. Group Analysis 1977; 10: S2-16.

28 Filstead WJ, Rossi JJ. Therapeutic milieu, therapeutic community, and milieu therapy: some conceptual and definitional distinctions. In The Therapeutic Community: A Sourcebook of Readings (eds JJ Rossi, WJ Filstead): 3-13. Behavioural Publications, 1973.

29 Hodgson R, Alwyn T, John B, Thom B, Smith A. The Fast Alcohol Screening Test. Alcohol Alcohol 2002; 37: 61-6.

30 Gavin DR, Ross HE, Skinner HA. Diagnostic validity of the drug abuse screening test in the assessment of DSM-III drug disorders. Br J Addict 1989; 84: 301-7.

31 Goldberg D. General Health Questionnaire (GHQ-12). Nfer-Nelson, 1992.

32 Tyrer $\mathrm{P}$, Nur U, Crawford M, Karlsen S, MacLean C, Rao B, et al. The Social Functioning Questionnaire: a rapid and robust measure of perceived functioning. Int J SOC Psychiatry 2005; 51: 265-75. 
33 Sorgi P, Ratey JJ, Knoedler DW, Markert RJ, Reichman M. Rating aggression in the clinical setting: a retrospective adaptation of the Overt Aggression Scale: preliminary results. I Neuropsychiatry Clin Neurosci 1991; 3: S52-56.

34 Larsen DL, Attkisson CC, Hargreaves WA, Nguyen TD. Assessment of client/ patient satisfaction: development of a general scale. Eval Program Plann 1979; 2: 197-207.

35 Rutter $D$, Tyrer $P$. The value of therapeutic communities in the treatment of personality disorder: a suitable place for treatment? J Psychiatr Pract 2003; 9: 291-302.

36 Pearce $S$, Autrique $M$. On the need for randomised trials of therapeutic community approaches. Ther Communities 2010; 31: 338-55.

37 Shaw C. External assessment of health care. BMJ 2001; 322: 851.

38 Worrall A. The service context for clinical guidelines: supporting guideline implementation by assuring and improving the quality of service in which clinicians work. Int Rev Psychiatry 2011; 23: 336-41.

39 Haigh R, Tucker S. Democratic development of standards: the community of communities - a quality network of therapeutic communities. Psychiatr $Q$ 2004; 75: 263-77.
40 Autrique M, Pearce S, Vanderplasschen W, Haigh R. The impact of a randomised trial on TC environments. Ther Communities 2015; 36: 137-44.

41 Moos R. Community Oriented Programs Environment Scale Manual. Mind Garden, 2003.

42 Crawford MJ, Price K, Gordon F, Josson M, Taylor B, Bateman A, et al. Engagement and retention in specialist services for people with personality disorder. Acta Psychiatr Scand 2009; 119: 304-11.

43 Pearce S, Haigh R. Mini therapeutic communities - a new development in the United Kingdom. Ther Communities 2008; 29: 111-24.

44 Mahlke $\mathrm{Cl}$, Bock T. Peer to peer support in severe mental disorders: affective disorders, psychosis and personality disorder - a randomized controlled trial. Eur Psychiatry 2015; 30: 165.

45 Baumeister RF, Leary MR. The need to belong: desire for interpersonal attachments as a fundamental human motivation. Psychol Bull 1995; 117: 497-529.

46 National Institute for Care and Health Excellence. Borderline Personality Disorder: Recognition and Management. NICE, 2015 (https://www.nice.org.uk/ guidance/cg78).

\section{poems by doctors}

\section{Article 3}

\section{Frederick Hopkinson}

She screams.

Seconds pass, she screams again.

Psst, psst, psst psst psstpsstpsst.

The cuff tightens around her arm.

Our grip on her tightens.

Stabbing. Holding her down, stabbing. Missing. Holding her down. Stabbing.

Blood. Bruises.

No answers.

She screams and screams and screams.

In protest? In pain?

Tap chest. Push tummy.

She screams and stares me in the eye.

She doesn't eat. She doesn't drink.

We won't let go.

She screams and screams.

Plaques? Tangles?

The cuff tightens.

An ambulance called. Looking for

something. New surroundings.

Fluids: lines that tie her to the earth.

No answers.

Back to the ward, familiar

surroundings?

Shouts. Screams. Falls. Bruises.

Stab.

The needle probes for answers.

Flesh, tendons, blood, bruises.

Finds nothing.

Flash.

Xrays penetrate, organs exposed.

Nothing found.

Shouts. Screams. Every few seconds screams

No rest.

Anger.

We search on

Grip. Tap. Prod. Stab. Flash. Scream. Screams, screams

No rest.

Anger.

We search on.

Grip. Tap. Prod. Stab. Flash. Scream. Screams, screams 